\title{
Finite elements analysis of an electrochemical coating process of an irregularly shaped cathode with COMSOL Multiphysics ${ }^{\circledR}$
}

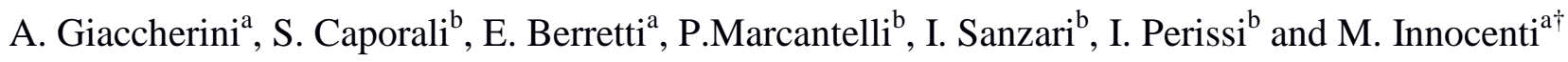 \\ $\dagger$ Active ECS member \\ a Dipartimento di Chimica, Università di Firenze, via della Lastruccia 3, 50019 Sesto Fiorentino, Firenze, ITALY \\ b Consorzio INSTM , via Giusti 9, 50121, Firenze, ITALY
}

\begin{abstract}
The overall goal of this work is the use of COMSOL Multiphysics ${ }^{\circledR}$ in the modelling of the current density distributions for the electrodeposition of Aluminum coatings from Ionic Liquids baths. The local current distribution is strongly dependent on the conductivity, on the distribution of concentrations and on the geometry of the galvanic cell, and, therefore, such a calculation can only be performed by the numerical solution of the Partial Differential Equations (PDE) governing the system. This work approach is to exploit computational techniques based on the wellknown Finite Elements Analysis (FEA) to obtain stationary and dynamical solutions of such a problem. The ability to predict the local current density on an electrode is crucial to eventually evidence portions where the deposition may be invalidated.
\end{abstract}

\section{Introduction}

The electroplating technology from aqueous solutions has some limitations. In fact, not all metal ions can be deposited from aqueous solution due to the small electrochemical window of water $(1,2)$. Recently, Ionic Liquids have been applied to the deposition of coatings with a high technological potential $(3,4)$. We refer to ionic liquids (ILs) speaking of "Room temperature molten salts", which have been in their infancy 15 years ago, and are now assessed as viable alternative to traditional electrochemical media, able to join the room temperature melting point of organic solvents with the optimal electrochemical behavior of molten salts(5). This paper presents the results of a Finite Elements Analysis (FEA) study to obtain the current distribution, which constitutes the driving force for the electrodeposition process of Aluminum from first generation ILs. In the field of FEA simulations, a common aim is to obtain reliable models in order to optimize the process setups "a priori", and we believe that this approach can be extended to electroplating industrial processes in order to estimate the most favorable geometry of the anodes in a galvanic cell(6). The main focus of this paper is the analysis of the concentration distribution at the electrodes boundaries and its dependence on the concentration. The calculation has been performed by means of the simulation program COMSOL Multiphysics ${ }^{\circledR}$.

\section{Governing differential equations system}

On a merely mathematical level, the evolution of an electrochemical system is defined by a systems of fundamental Partial Differential Equations (PDE). Previous studies approached this modelling problem taking into account only the primary current 
distribution (7). This approach resulted suitable to develop a model able to account for the time-dependent evolution of the current density distribution, which can be related to the change of the concentration distribution in the system. In the present study we chose to develop a model based on the tertiary current distribution (8).

First of all, the ohm's law, equation 1, takes into account for the electrical behavior of an electrochemical system (9-13).

$$
\nabla^{2} \phi_{l}=\frac{-Q_{l}}{\sigma_{l}}
$$

Where $\phi_{l}$ is the electric potential in the electrolyte; $\sigma_{l}$ is the conductivity of the electrolyte and $Q_{l}$ is the electric charge. The Nerst-Plank equation describes the flux of the chemical species due to general transport phenomena including convection, diffusion and migration (eqn. 2).

$$
\underline{N_{i}}=-D_{i} \nabla c_{i}-z_{i} u_{m, i} F c_{i} \nabla \phi_{l}+\underline{u} c_{i}
$$

Where $\mathrm{c}_{\mathrm{i}}, \mathrm{D}_{\mathrm{i}}, \mathrm{u}_{\mathrm{i}}$, and $\mathrm{z}_{\mathrm{i}}$ are the concentration, the diffusion coefficient, the mobility and the charge of the of the $i_{\text {th }}$ species, respectively; $F$ the Faraday's constant; $\underline{u}$ the velocity field; $R_{i}$ the reaction term for $A$ and $B$.

The Dirichlet problem is completely defined with boundary condition such as electroneutrality, which holds for the bulk electrolyte (eqn. 3).

$$
\sum_{i} z_{i} c_{i}=0
$$

Other conditions are related to the current at the boundary (eqns 4-6).

$$
\begin{gathered}
\underline{i}_{l} \cdot \underline{n}=i_{\text {total }} \\
i_{\text {total }}=\sum_{i} i_{l o c, m}+i_{d l} \\
i_{l o c}=i_{0}\left(\frac{C_{R}}{C_{R}^{0}} e^{\frac{\alpha_{a} F \eta}{R T}}-\frac{C_{O}}{C_{O}^{0}} e^{\frac{-\alpha_{c} F \eta}{R T}}\right)
\end{gathered}
$$

where, $\underline{i}_{l}$ is the current distribution vector; $i_{d l}$ is the capacitive current density; $i_{l o c, m}$ is the faradaic current density for the $\mathrm{m}_{\mathrm{th}}$ process; $i_{0}$ is the exchange current; $C_{R} / C_{R}^{0}$ is the ratio of the concentration to the bulk concentration; $\alpha_{a}$ is the electron transfer coefficient; $R$ is the perfect gasses constant; $T$ is the temperature and $\eta$ is the electrode overpotential defined by equation 7 .

$$
\eta=\phi_{l}+\phi_{s}-E_{e q}
$$

Here, $\phi_{l}+\phi_{s}$ is the electrical potential drop across the electrode interface. In order to calculate the change in composition of the electrolyte near the electrodes due to electron 
transfer, it's well established the validity of the following Faraday equation (eqn. 8):

$$
R_{i, m}=\frac{-v_{i, m} i_{l o c, m}}{n_{m} F}
$$

\section{$\underline{\text { FEA with COMSOL Multiphysics }}{ }^{\circledR}$}

Recently, COMSOL Multiphysics ${ }^{\circledR}$ has implemented different mathematical approaches to model electrochemical systems. In particular, the electrodeposition module is flexible enough to allow the solution of the system by means of several different approximations widely used in the field of galvanic or potentiostatic process engineering. They are the following: Primary current distribution, which solves the PDE system without the Nerst-Plank equation for the transport phenomena and without the ButlerVolmer equation for the boundary conditions on the electrodes surfaces. (No faradaic process); Secondary current distribution, which solves the PDE system without the NerstPlank equation for the transport phenomena and with the Butler-Volmer equation for the boundary conditions on the electrodes surfaces. (No transport phenomena); Tertiary current distribution, which solves the complete PDE system shown in the last paragraph (13-16).

Furthermore, the program allows to use different approximations for the Butler-Volmer equation [6]. In the case of a negligible transport in the bulk electrolyte respect to the electron transfer process the ratio $C_{O} / C_{O}^{0}=1$, so that the local current can be described by equation 9.

$$
i_{l o c}=i_{0}\left(e^{\frac{\alpha_{a} F \eta}{R T}}-e^{\frac{-\alpha_{c} F \eta}{R T}}\right)
$$

When $\eta \approx 0$ the exponential term in the Butler-Volmer can be linearized (eqn. 10):

$$
i_{\text {loc }}=i_{0}\left(\frac{C_{R}}{C_{R}^{0}} \frac{\alpha_{a} F \eta}{R T}+\frac{C_{O}}{C_{O}^{0}} \frac{\alpha_{c} F \eta}{R T}\right)
$$

When $\eta \rightarrow+\infty$ we obtain the anodic tafel equation (eqn. 11):

$$
i_{\text {loc }}=\frac{C_{R}}{C_{R}^{0}} e^{\frac{\alpha_{a} F \eta}{R T}} i_{0}
$$

With $\eta \rightarrow-\infty$ we obtain the cathodic tafel equation (eqn. 12):

$$
i_{l o c}=\frac{C_{O}}{C_{O}^{0}} e^{\frac{-\alpha_{c} F \eta}{R T}} i_{0}
$$

For the case in study, we used a tertiary current distribution with both Butler-Volmer equation and Butler-Volmer linearized equation as boundary conditions for the electrodes surfaces. 


\section{Results}

Two different problem has been investigated, a 2D system, constituted by one cathode located between two anodes and a 3D system constituted by one cathode, irregularly shaped, located between two anodes.

The first system has been used to understand the different possibilities available in the electrodeposition module of COMSOL Multiphysics ${ }^{\circledR}$ while applying different types of approximation. The actual problem will be useful to compare the theoretical results with experimental data to validate the model approximation. We chose to simulate a potentiostatic deposition of Aluminium from 1-Butyl-3-methylimidazolium (BMIm) $\mathrm{Al}_{2} \mathrm{Cl}_{7}$, with an applied voltage of $2 \mathrm{~V}$.

\section{$\underline{2 \mathrm{D} \text { case }}$}

The simple 2D geometry (Fig. 1) was employed to optimize the parameters and the settings that will be implemented in the following actual 3D model.

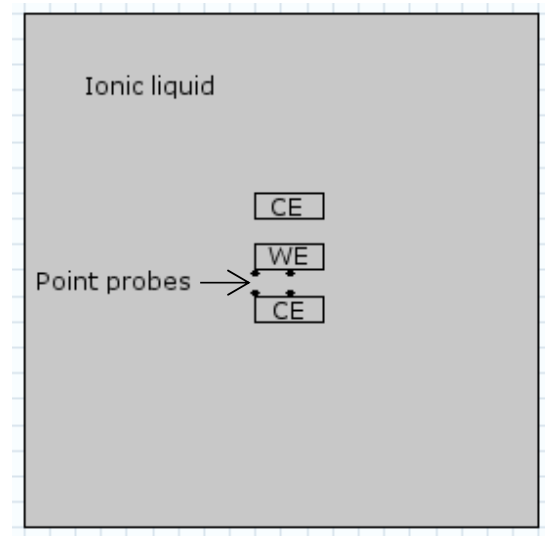

Figure 1. Scheme showing the electrodes geometry considered for the 2D system.

We used a fine triangular mesh (built by COMSOL Multiphysics ${ }^{\circledR}$ ), using the tertiary current distribution mode and different settings for the Butler-Volmer equation.

Four cases were numerically solved and the results compared: linearized Butler-Volmer equation (eq. 10); linearized Butler-Volmer equation without pre exponential factors (eqns. 9, 10); Butler-Volmer equation without pre exponential factors (eqn. 9) and complete Butler-Volmer equation (eqn. 6). Due to their different dynamics, different time scales have been applied; in all the cases, we consider that the process has ended when the current distribution (or the electrolyte potential) do not change anymore. For cases 1 and 3 this condition is reached after 30 minutes; 30 seconds and 20 minutes are required for cases 2 and 4, respectively.

The numerical results of cases 1, 2, 3 and 4 are compared in terms of concentration variation as "measured" by virtual point probe on the edge of the electrode, as shown in Figure 1. The numerical results of case 4 are shown in Figure 3. The variation of 
concentration at cathode shows that for cases 1 and 3 , the concentration of $\mathrm{AlCl}_{4}{ }^{-}$ decreases down to negative values (the starting value is $30 \mathrm{~mol} \mathrm{~m}^{-3}$ ). This has no physical meaning and, therefore, these results must be discarded. Cases 2 and 4 have a much more fast evolution, reaching the stationary state in a few minutes or less. At the cathode, the variations cannot reach the complete depletion of the electroactive species due to the pre exponential terms in the fully coupled models (cases 2 and 4). This counterintuitive behavior can be explained in terms of transport phenomena. In case 2 the concentration of the oxidate species at the cathode increases after 5 seconds, and in case 4 the concentration of such species oscillates. It seems reasonable to think that the difference of the current density on the edge with respect to the central part of the electrode accounts for this behavior.

The current density results higher on the edge, and this leads to a depletion of the electroactive species in the proximity of the edge. This depletion establishes a concentration gradient, parallel to the electrode surface, from the center of the surface to the edge; consequently, a diffusive flow is established and the concentration at edge increases. In the meanwhile, the current decreases; consequently, the depletion rate of the electroactive species decreases and, temporary, the tangential flux can compensate for the depletion. In the model the depletion slows down, dramatically changing the electrolyte potential even leading to reverse the sign of the electrode overpotential.

The variation of $\mathrm{BMIm}^{+}$cation concentration is mainly due to the electroneutrality condition, coupled with the transport phenomena. However, as shown in Figure 2, these strange trends are not present in the central probes; we can guess that this counterintuitive behavior can be mainly due to geometrical effects on the concentration in the tertiary current distribution.
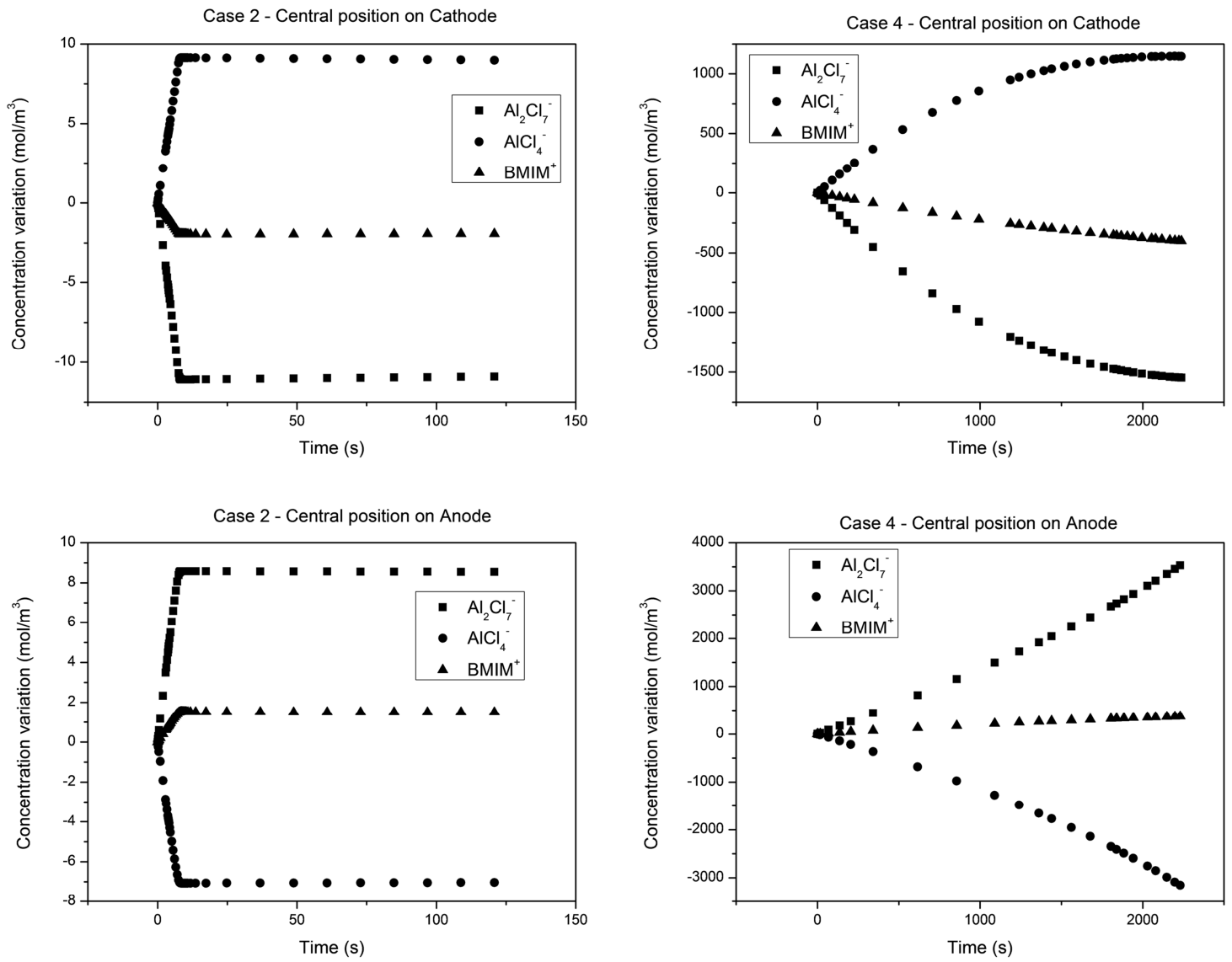
Figure 2. Concentration evolution on the point probes for cases 2 and 4
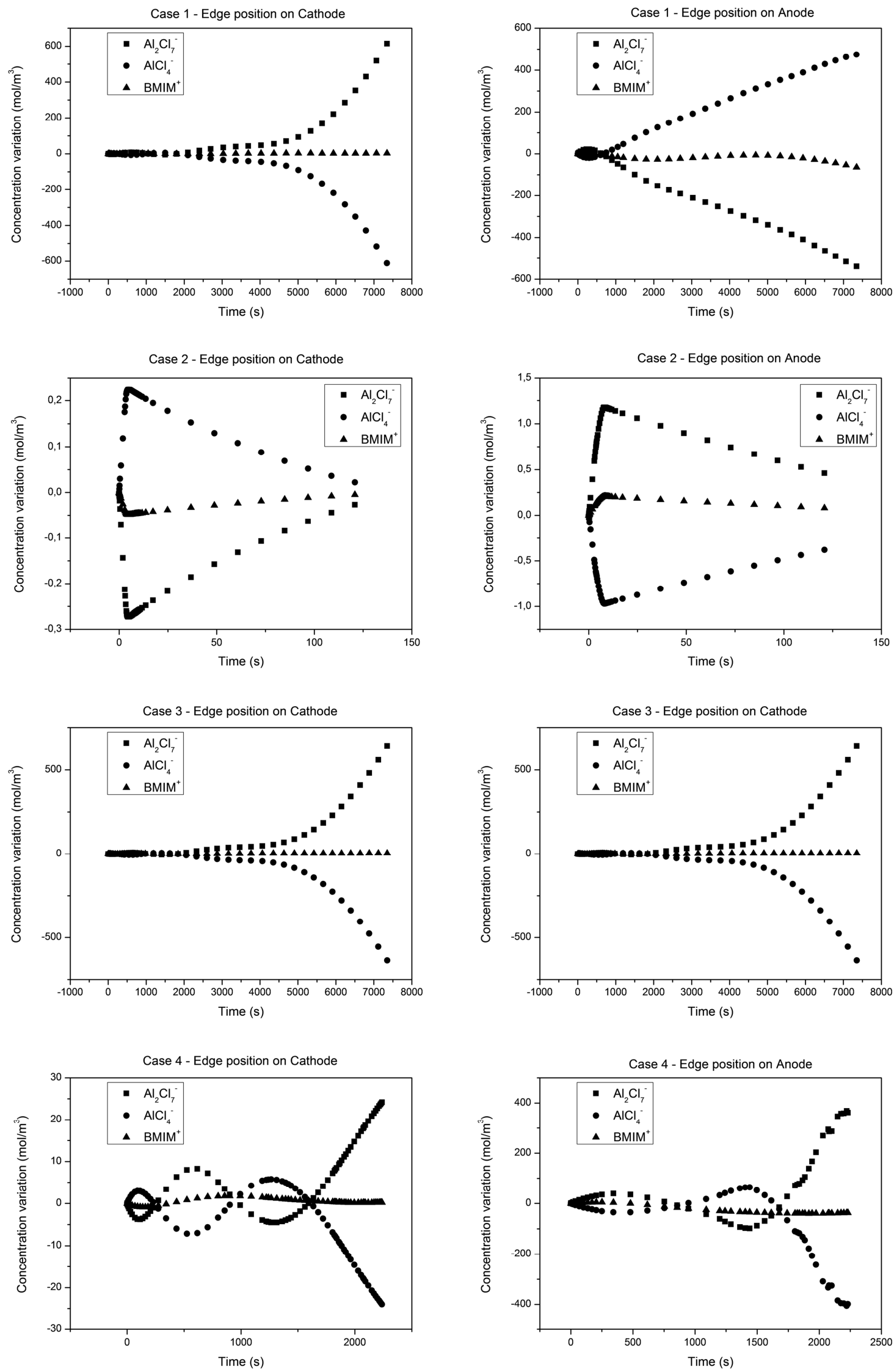
Figure 3. Concentration evolution on the edge probes

\section{$\underline{3 \mathrm{D} \text { case }}$}

The actual problem is the potentiostatic coating of a complex-shaped cathode (Figure 4 ). For the modeling purposes, the large faces have been insulated and the simulation protracted up to one minute.

Peculiar features stumbled out from the solution of this system that will be used for the future development of the electrodeposition modeling. In particular, analyzing the electrolyte potential and current distribution after 30 seconds, the maximum current density is located at the bottom of the "valleys" and not on the "pointy" edges as was expected. Further studies will be carried on to understand the reason for this counterintuitive distribution.

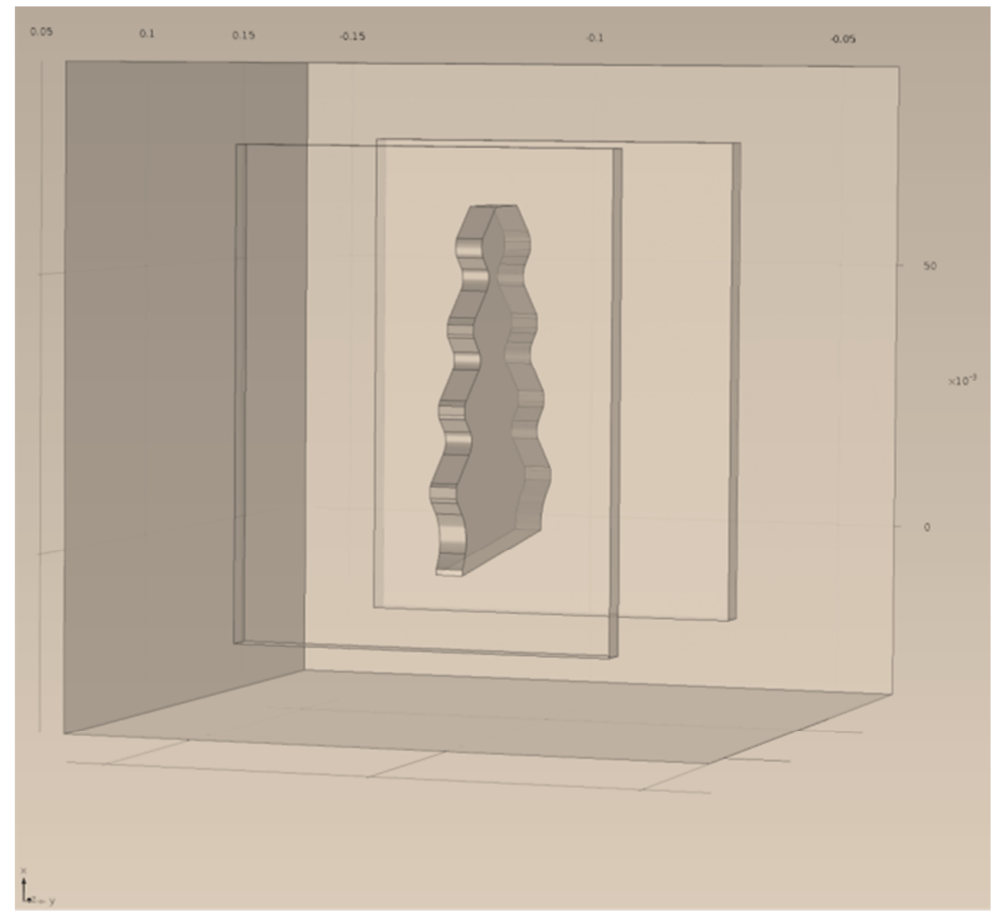

Figure 4. Geometry of the virtual electrodeposition cell

However, these results evidenced the different evolutions of the concentration of the electroactives chemical species during the process, and how these differences affect the resulting current density distribution. Counterintuitive changes of the concentration on the edges of the 2D model have been shown; these can be related to a diffusion flux parallel to the electrode boundary. This effect have been reproduced in a 3D model, irregularly shaped, obtaining temporary higher current density on the "valleys" and not on the "peaks". The modelling strategy developed can be used to account for the effects of the concentration on the electrodeposition process.

\section{Acknowledgments}

"The research leading to these results has received funding from the European Union Seventh Framework Programme (FP7/2007- 2013) under grant agreement $n^{\circ} 608698$ " 


\section{References}

1. M. Armand, F. Endres, D. R. MacFarlane, H. Ohno and B. Scrosati, Nature Materials, 8, 621, (2009).

2. F. Endres, Elctrodeposition from Ionic Liquid, Wiley-VCH, (2008)

3. Q.X. Liu, S. Zein El Abedin and F. Endres, Surf. Coat. Technol., 201, 1352, (2006).

4. S. Takahashi, N. Koura, S. Kohara, M.-L. Saboungi, L.A. Curtiss, Plasmas and Ions, 2, 91, (1992).

5. T. Jiang, M.J. Chollier Brym, G. Dubé, A. Lasia, G.M. Brisard, Surf. Coat. Technol, 201, 1, (2006).

6. R. Li, L. Fang, Y. Deng, S. Liu, ICCDA, Nanjing, (2010).

7. I. Perissi, C. Borri, S. Caporali, and A. Lavacchi, "Current Density Simulations in the Electrodeposition from Ionic Liquids: Effects of the Conductivity", COMSOL Conference, Milan, (2009).

8. A.Lavacchi, U. Bardi, C. Borri, S. Caporali, A. Fossati, I. Perissi, J. Appl. Electrochem., (2009).

9. G. Richardson and J.R. King, J. of Math. Eng., 59, 239, (2007).

10. C.T.J. Low, E.P.L. Roberts, F.C. Walsh, Electrochim. Acta., 52, 3831, (2007).

11. D. Britz, Digital simulations in electrochemistry, Springer, (2005).

12. T.J. Davies, C.E. Banks, R.G. Compton, J. Solid State Electrochem., 9,797, (2005).

13. W. Lu, X. Wang, X. Li, Y. Yin, Z. You, J. Nanoeng. Nanosyst., 228, 93, (2014).

14. E.J.F. Dickinson, H. Ekstrom, Ed. Fontes, Electrochem. Commun., 40, 71, (2014).

15. V. Ionescu, Romanian J. Phys., 59, 285, (2014).

16. J. Vaughan and D. Dreisinger, ECS Trans., 16, 397 (2009).

17. A. Bonnefont, F. Argoul, M.Z. Bazant, J. Electroanal. Chem., 550, 52, (2001).

18. J. Tang, K. Azumi, Electrochim. Acta., 56, 1130, (2011). 